UCSBTH-94-15

hep-th/9405070

\title{
Timelike Duality
}

\author{
Dean L. Welch \\ Department of Physics \\ University of California \\ Santa Barbara, CA 93106-9530 \\ dean@cosmic.ucsb.edu
}

\begin{abstract}
Several stationary solutions of the low energy string equations are dualized with respect to their timelike symmetry. Many of the duals have simple physical interpretations. Those of the nonextremal three dimensional black hole and black string are negative mass black strings. The extremal cases of these, and extremal higher dimensional black strings also, give negative energy plane fronted waves. In fact, all of the duals of positive mass solutions that will be considered here have nonpositive energies, but an argument is given which suggests that this is not true in general.
\end{abstract}

\section{Introduction}

One of the interesting properties of string theory is that it has a symmetry called duality. The simplest example of duality comes from considering a flat spacetime with one dimension compactified to form a circle of radius $R$. For a theory based on strings this solution is equivalent to one where the compactified direction has radius $1 / R$ [1]. However, duality is much more general than this. There exists a more general duality transformation that maps any solution of the low energy equations that has a symmetry to another solution of the low energy equations [2]. In general these solutions will have very different geometries. If this symmetry is spacelike and has compact orbits then both 
solutions correspond to the same conformal field theory and so are equivalent as string theory solutions [3].

Although duals are usually taken with respect to spacelike symmetries, when a spacetime is stationary one can take a dual with respect to its timelike symmetry. As is the case for a spacelike symmetry, dualizing with respect to a timelike symmetry maps one solution of the low energy string equations to another. It is currently not known when these solutions are equivalent as string theory solutions. The two dimensional black hole [4] [5] has been dualized with respect to its timelike symmetry and this provides an example of when they are equivalent (another argument that this will be true in more general circumstances will be presented shortly). This duality transformation maps a solution with positive mass and a horizon to one with a negative mass naked singularity. It has been shown that these dual solutions arise from different gaugings of the same WZW model, therefore they correspond to the same conformal field theory and are equivalent as string theory solutions. In fact the two dimensional black hole is its own dual in the sense that this duality transformation just exchanges parts of the Penrose diagram, the part behind the singularity is exchanged with the asymptotically flat part.

It is well known that when we dualize a solution with respect to a symmetry that has a fixed point the dual solution will have a singular geometry. This is most easily seen by dualizing flat spacetime written in cylindrical coordinates [3]. If we take the metric to be

$d s^{2}=-d t^{2}+d r^{2}+r^{2} d \theta^{2}$, dualizing with respect to $\theta$ gives $\widetilde{d s}^{2}=-d t^{2}+d r^{2}+r^{-2} d \theta^{2}$ (the tilde symbol will be used to denote dual fields). The dual metric is singular at the fixed point of $\theta, r=0$. However, it is nonsingular as a solution to string theory [3], as it must be since it is the dual of a nonsingular solution.

The symmetry that we dualizing with respect to need not have a fixed point to get such a singularity, it is sufficient for it to become null. If $t$ is the timelike coordinate at infinity then we know that $g_{t t}=0$ at, or outside of, an event horizon. The dual solution will be singular where this happens. If a black hole has nonzero rotation then $g_{t t}$ will be zero on the boundary of the ergosphere, which is outside of the horizon.

The low energy string action is

$$
S=\int d^{n} x \sqrt{-g} e^{-2 \phi}\left[R+4(\nabla \phi)^{2}-\frac{1}{12} H_{\alpha \beta \gamma} H^{\alpha \beta \gamma}+a\right]
$$


Where, $a$ is a centeral charge term, $R$ is the Ricci scalar constructed from the spacetime metric, $H_{\alpha \beta \gamma}$ is a totally antisymmetric three tensor, and $\phi$ is a scalar field called the dilaton. The equations of motion that follow from this action are

$$
\begin{aligned}
& R_{\alpha \beta}+2 \nabla_{\alpha} \nabla_{\beta} \phi-\frac{1}{4} H_{\alpha \sigma \gamma} H_{\beta}^{\sigma \gamma}=0 \\
& \nabla^{\alpha}\left(e^{-2 \phi} H_{\alpha \beta \gamma}\right)=0 \\
& 4 \nabla^{2} \phi-4(\nabla \phi)^{2}+a+R-\frac{1}{12} H^{2}=0
\end{aligned}
$$

Just applying the usual duality transformation [2] immediately presents a problem with the transformation of the dilaton. Simply applying the usual transformation gives $\tilde{\phi}=\phi-1 / 2 \ln g_{t t}$, but $g_{t t}<0$ outside the horizon. To make sense of this we can Euclideanize the metric by taking $t \rightarrow i \tau$ and then dualize with respect to the Euclidean time, $\tau$, giving a dual dilaton of $\tilde{\phi}=\phi-1 / 2 \ln g_{\tau \tau}=\phi-1 / 2 \ln \left(-g_{t t}\right)$. Finally, after dualizing we can continue back to a Lorentzian metric. The fact that the off diagonal terms, $g_{t j}$ and $B_{t j}$ (as usual $i, j, k, \ldots$ are indicies other than $t$ ), temporarily become complex presents no problem, since they become real when we continue back. We can either just leave them complex while we dualize or we can continue other parameters to imaginary values to keep them real.

Applying the above procedure of analytically continuing, dualizing, and then continuing back we find that given a solution of the low energy string equations that is independent of time, $\left(g_{\alpha \beta}, B_{\alpha \beta}, \phi\right)$, then $\left(\tilde{g}_{\alpha \beta}, \tilde{B}_{\alpha \beta}, \tilde{\phi}\right)$ is also a solution where 2]

$$
\begin{aligned}
\tilde{g}_{t t} & =1 / g_{t t}, \quad \tilde{g}_{t i}=-B_{t i} / g_{t t} \\
\tilde{g}_{i j} & =g_{i j}-\left(g_{t i} g_{t j}-B_{t i} B_{t j}\right) / g_{t t} \\
\tilde{B}_{t i} & =-g_{t i} / g_{t t}, \quad \tilde{B}_{i j}=B_{i j}-2 g_{t[i} B_{j] t} / g_{t t} \\
\tilde{\phi} & =\phi-\frac{1}{2} \ln \left(-g_{t t}\right)
\end{aligned}
$$

Notice that the $t j$ components of the dual metric and antisymmetric tensor have signs that are different from what one might have expected (this can be undone by changing the definitions of the signs of the antisymmetric tensor potential).

It was proven by Roček and Verlinde that when a spacelike symmetry that we are dualizing with respect to has compact orbits then the original solution and its dual correspond to the same conformal field theory [3]. The solutions that we are dualizing have horizons. When a solution has a horizon its Euclidean time must be identified with some 
finite period to avoid a canonical singularity. Recall that we obtained our duality transformation by continuing to Euclidean time and then continuing back after making the usual duality transformation. The requirement of having a periodic Euclidean time, together with the results of Roček and Verlinde, tell us that the two Euclidean solutions correspond to the same conformal field theory. This suggests that their Lorentzian signature counterparts also correspond to the same conformal field theory. This provides a second, more general, argument that they do. Some evidence for this will be presented later.

In this work some stationary solutions to the low energy string equations that have horizons will be dualized with respect to their timelike symmetry. The solutions we will consider are: the three dimensional black hole [6] [7] [8], the three dimensional black string [9] [10], and black strings in five or more dimensions [10] (which effectively includes black holes in four or more dimensions). For all these solutions, except the three dimensional black hole with nonzero rotation, $g_{t t}=0$ on the horizons, as stated before this tells us that the duals have naked singularities there. The dual of the rotating three dimensional black hole also has a naked singularity, but it is where the boundary of the ergosphere of the original solution was. The tangent to the horizon of a three dimensional black hole is a Killing vector that is null on the horizon. One might think it is more natural to dualize the three dimensional black hole with respect to this symmetry so that the dual solution is singular on what was the horizon of the original solution. This dual of the three dimensional black hole seems more similiar to the other dual solutions and will also be taken.

If in addition we consider spacetimes that are asymptotically flat and that have a spacelike translational symmetry in addition to their timelike symmetry, then we can establish two general results. It has already been proven [10 that if we dualize an asymptotically flat spacetime with respect to the spacelike translational symmetry then the momentum along the symmetry direction per length, called $P_{x}$ here, will be interchanged with the charge of the $H_{\alpha \beta \gamma}$ per length, denoted $Q$. In other words we have $\tilde{P}_{x}=Q$ and $\tilde{Q}=P_{x}$. When we take one of the these spacetimes that is stationary and dualize with respect to the time we get a similiar result that differs only by a minus sign, we get $\tilde{P}_{x}=-Q$ and $\tilde{Q}=-P_{x}$.

In all the cases we will consider the dual solutions of positive mass solutions will have nonpositive energy. Some of the dual solutions are plane fronted waves, these have zero rest mass and travel at the speed of light. In these cases one would only talk about the energy of the solution. Only the dual of the usual four dimensional black hole, or equivalently 
the of five dimensional black string, had zero mass. The other duals had masses that were negative. This suggests a general result that dualizing a positive mass solution with respect to a time gives a solution that has nonpositive energy. It is not known if this is true, although an argument is presented that suggests it is not. A weaker result concerning the behavior of the energy of the dual solutions will be established.

\section{The Dual Solutions}

\subsection{The Three Dimensional Black String}

The first solution we will dualize is the three dimensional black string [9]. It is given by

$$
\begin{gathered}
d s^{2}=-\left(1-\frac{M}{r}\right) d t^{2}+\left(1-\frac{Q^{2}}{M r}\right) d x^{2}+\left(1-\frac{M}{r}\right)^{-1}\left(1-\frac{Q^{2}}{M r}\right)^{-1} \frac{l^{2} d r^{2}}{4 r^{2}} \\
\phi=-\frac{1}{2} \ln r l, \quad B_{x t}=\frac{Q}{r}
\end{gathered}
$$

Where $M$ is the mass per length of the string, $Q$ is the charge of the $H_{\alpha \beta \gamma}$ field per length of the string, and $l$ is related to the central charge. In addition to the symmetry of translating along $t$ there is also a spatial translational symmetry in the $x$ direction. This solution has an event horizon at $r=M$ (for $Q^{2} \leq M^{2}$ ) and if $Q \neq 0$ there is also an inner horizon at $r=Q^{2} / M$. In the extremal case, $Q^{2}=M^{2}$, there is only the event horizon. One way to obtain this solution is to take the product of the two dimensional black hole [4] and a translation, this gives us the uncharged three dimensional black string. To add charge we can boost this solution and then dualize on $x$ [10].

Applying (1.3) to this gives

$$
\begin{aligned}
\tilde{d s}^{2}=\left(1-\frac{M}{r}\right)^{-1} & {\left[-d t^{2}-2 \frac{Q}{r} d x d t+\left(1-\frac{M}{r}\left(1+\frac{Q^{2}}{M^{2}}\right)\right) d x^{2}\right] } \\
& +\left(1-\frac{M}{r}\right)^{-1}\left(1-\frac{Q^{2}}{M r}\right)^{-1} \frac{l^{2} d r^{2}}{4 r^{2}} \\
\tilde{\phi} & =-\frac{1}{2} \ln l(r-M), \quad \tilde{B}_{\alpha \beta}=0
\end{aligned}
$$

Although this may not look familiar it is just a boosted chargeless negative mass black string. As we will see in the next section the fact that it is boosted is a manifestation of the effect dualizing has on the charge and momentum of an asymptotically flat solution 
that also has a spacelike symmetry. What was $H_{\alpha \beta \gamma}$ field charge in the original solution is now momentum along $x$. To see that this is a chargeless negative mass black string we can boost, and shift $r$, by the coordinate transformation

$$
t=\left(1-\frac{Q^{2}}{M^{2}}\right)^{-1 / 2}\left(\hat{t}-\frac{Q}{M} \hat{x}\right), \quad x=\left(1-\frac{Q^{2}}{M^{2}}\right)^{-1 / 2}\left(\hat{x}-\frac{Q}{M} \hat{t}\right), \quad \hat{r}=r-M
$$

In these coordinates the solution is

$$
\begin{gathered}
\widetilde{d s}^{2}=-\left[1+\frac{M}{\hat{r}}\left(1-\frac{Q^{2}}{M^{2}}\right)\right] d \hat{t}^{2}+d \hat{x}^{2}+\left[1+\frac{M}{\hat{r}}\left(1-\frac{Q^{2}}{M^{2}}\right)\right]^{-1} \frac{l^{2} d \hat{r}^{2}}{4 \hat{r}^{2}} \\
\tilde{\phi}=-\frac{1}{2} \ln \hat{r} l, \quad \tilde{B}_{\alpha \beta}=0
\end{gathered}
$$

This is an uncharged black string with mass per length $-M\left(1-Q^{2} / M^{2}\right)$.

We can also anticipate this result from conformal field theory [11]. We know that the three dimensional black string can be obtained by gauging an axial symmetry of a WZW model [9]. Suppose we have a WZW model with a symmetry group, $G$, and we want an axial gauging of some Abelian subgroup of $G$, say $H$. Then the action of this on an element of $G$, say $g$, is $g \rightarrow h g h$, where $h$ is an element of $H$. For $h$ close to the identity element we get the infinitesimal version of this, $\delta g=\epsilon g+g \epsilon$. The action for a vector gauging is $g \rightarrow h g h^{-1}$, the infinitesimal version of which is $\delta g=\epsilon g-g \epsilon$.

If we take a WZW model with $G=S L(2, R)$ and axially gauge a certain one dimensional subgroup of $G$ we will get a two dimensional black hole [4] [5] [9]. If we again take $G=S L(2, R)$, but now vector gauge this subgroup we will get the dual (we are dualizing with respect to time since it is the only symmetry for these black holes) of the two dimensional black hole. This dual has a negative mass, however the two dimensional black hole is its own dual in the sense that what we have done by dualizing this solution amounts to exchanging parts of the Penrose diagram. The exchange is between the asymptotically flat part that has the horizon and the part behind the singularity [5] [4].

Adding one free boson $x$ to the action is equivalent to taking $G=S L(2, R) \times R$. If we do this and take the axial gauging of the same subgroup as in the two dimensional black hole case, and also allowing the translations to be gauged, we will get the three dimensional black string [9]. Suppose that we now take the same group, $G=S L(2, R) \times R$, and do a vector gauging of the same subgroup. Since we now have $\delta g=\epsilon g-g \epsilon$ we expect the $R$ to be unaffected by the gauging because it is Abelian. We will then have a product of 
a vecor gauged $S L(2, R)$ and $R$, which is an uncharged negative mass three dimensional black string.

In addition to showing us that dualizing this solution gives the product of a negative mass two dimensional black hole and $R$, it also gives us the origin of the negative mass. Following the example of dualizing the two dimensional black hole, we can interpret this duality as mapping the exterior part of the black string to the product of $R$ with the part of the two dimensional black hole that is behind the horizon. It also shows us that these solutions correspond to the same conformal field theory since they are just different gaugings of the same WZW model.

The extremal black string solution is (2.1) with $Q=M$, the dual of this is (2.2) with $Q=M$. This metric can no longer be diagonalized, but if we make the coordinate transformation $u=t+x, v=t-x$, and $R=\frac{l}{2} \ln (r-M)$, then the solution is in the form

$$
\begin{aligned}
\widetilde{d s} & =-d u d v-M e^{-2 R / l} d u^{2}+d R^{2} \\
\tilde{\phi} & =-\frac{R}{l}-\frac{1}{2} \ln l, \quad \tilde{B}_{\alpha \beta}=0
\end{aligned}
$$

This is just a plane fronted wane in the precence of a linear dilaton [10]. Using the mass formula from [10] (for the specific form of the mass formula see section 3), which really gives us the energy of the solution, shows that this has a negative energy.

It is interesting to compare these results with what we get by taking a three dimensional black string and dualizing it with respect to some spacelike symmetry. For the nonextremal case dualizing it with respect to $x$ gives an uncharged boosted black string, with velocity depending on $\mathrm{Q}$ [10]. We get the three dimensional black hole (to be discussed below) by dualizing with respect to $\partial / \partial t+\partial / \partial x[7]$. This symmetry is spacelike everywhere outside of the horizon, but it becomes null at infinity. If we dualize the extremal three dimensional black string with respect to $x$ we get a positive energy plane fronted wave. To summarize, for the three dimensional black string dualizing with respect to $t$ gives negative energy versions of the same solutions as dualizing with respect to $x$ does.

\subsection{The Three Dimensional Black Hole}

The three dimensional black hole solution is [6] [7]

$$
\begin{gathered}
d s^{2}=-\frac{1}{l^{2}}\left(r^{2}-r_{+}^{2}-r_{-}^{2}\right) d t^{2}-\frac{2 r_{+} r_{-}}{l} d t d \theta+r^{2} d \theta^{2}+\frac{l^{2} r^{2} d r^{2}}{\left(r^{2}-r_{+}^{2}\right)\left(r^{2}-r_{-}^{2}\right)} \\
\phi=0, \quad B_{t \theta}=-\frac{r^{2}}{l}
\end{gathered}
$$


The mass of this black hole is $M_{b h}=\left(r_{+}^{2}+r_{-}^{2}\right) / l^{2}$ and its angular momentum is $J=$ $2 r_{+} r_{-} / l$, without loss of generality $J \geq 0$ is being taken.

This metric is asymptotically anti-de Sitter. It has two horizons, an event horizon located at $r=r_{+}$and an inner horizon at $r=r_{-}$. This solution has many of the features one usually associates with a black hole, in addition to the horizons it has an ergosphere and a nonzero Hawking temperature. However, it also has some unusual features for a black hole, its curvature is constant, $l$ is related to the curvature by $R_{\alpha \beta}=-\left(2 / l^{2}\right) g_{\alpha \beta}$, this also tells us it has no curvature singularity (when $J=0$ it has a Taub-NUT type singularity, if $J \neq 0$ it is singularity free). Unlike the three dimensional black string this has two distinct extremal limits, $J^{2}=l^{2} M^{2} \neq 0$ and $J=M=0$.

The nonextremal solution, $J^{2}<l^{2} M^{2}$, will be considered first. Applying (1.3) yields

$$
\begin{aligned}
& \widetilde{d s}^{2}=\frac{l^{2}}{r^{2}-r_{+}^{2}-r_{-}^{2}} {\left[-d t^{2}-2 \frac{r^{2}}{l} d t d \theta-\frac{r^{2}\left(r_{+}^{2}+r_{-}^{2}\right)-r_{+}^{2} r_{-}^{2}}{l^{2}} d \theta^{2}\right] } \\
&+\frac{l^{2} r^{2} d r^{2}}{\left(r^{2}-r_{+}^{2}\right)\left(r^{2}-r_{-}^{2}\right)} \\
& \tilde{\phi}=-\frac{1}{2} \ln \frac{r^{2}-r_{+}^{2}-r_{-}^{2}}{l^{2}}, \quad \tilde{B}_{t \theta}=-\frac{l r_{+} r_{-}}{r^{2}-r_{+}^{2}-r_{-}^{2}}
\end{aligned}
$$

Notice that this becomes singular at $r^{2}=r_{+}^{2}+r_{-}^{2}$, this is at what was the boundary of the ergosphere and occurs before we reach the horizon of the original solution. We now have not only $\partial / \partial t$ being timelike, but $\partial / \partial \theta$ being timelike as well. However, the signature of the dual spacetime is the same as the original, as will be seen shortly.

If we make the coordinate transformation

$$
t=\frac{r_{+}^{2} \hat{x}-r_{-}^{2} \hat{t}}{l \sqrt{r_{+}^{2}-r_{-}^{2}}}, \quad \theta=\frac{\hat{t}-\hat{x}}{\sqrt{r_{+}^{2}-r_{-}^{2}}}, \quad l \hat{r}=r^{2}-r_{+}^{2}-r_{-}^{2}
$$

Then these fields (2.7) become

$$
\begin{gathered}
\widetilde{d s}^{2}=-\left(1+\frac{r_{+}^{2}}{l \hat{r}}\right) d \hat{t}^{2}+\left(1+\frac{r_{-}^{2}}{l \hat{r}}\right) d \hat{x}^{2}+\left(1+\frac{r_{+}^{2}}{l \hat{r}}\right)^{-1}\left(1+\frac{r_{-}^{2}}{l \hat{r}}\right)^{-1} \frac{l^{2} d \hat{r}^{2}}{4 \hat{r}^{2}} \\
\tilde{\phi}=-\frac{1}{2} \ln \frac{\hat{r}}{l}, \quad \tilde{B}_{\hat{t} \hat{x}}=\frac{r_{+} r_{-}}{l \hat{r}}
\end{gathered}
$$

Comparing this with (2.1) we see that this is a black string with $M=-r_{+}^{2} / l$ and $Q=$ $-r_{+} r_{-} / l$. We also have time being periodic, since $\theta$ is periodic, so we must go to a covering space to avoid closed timelike curves. 
Now the first extremal case will be considered, $M=J=0$. Taking (2.7) with $r_{+}=$ $r_{-}=0$ and making the coordinate transformation $u=t, v=2 l \theta, R=l \ln r$, we have

$$
\begin{gathered}
\widetilde{d s}^{2}=-l^{2} e^{-2 R / l} d u^{2}-d u d v+d R^{2} \\
\tilde{\phi}=-\frac{R}{l}-\ln l, \quad \tilde{B}_{\alpha \beta}=0
\end{gathered}
$$

Which is again a plane fronted wave in the presence of a linear dilaton, it also has negative energy.

Now consider the other extremal case, $J=M l \neq 0$. This corresponds to inserting $r_{+}=$ $r_{-} \neq 0$ into (2.7). Doing this and making the coordinate change, $t=u-v r_{+}^{2} / 2 l^{2}, \theta=v / 2 l$, and $l R=r^{2}-2 r_{+}^{2}$, the metric becomes

$$
\widetilde{d s}^{2}=-\frac{l}{R} d u^{2}-\left(1+\frac{r_{+}^{2}}{l R}\right) d u d v+\frac{l^{2} d R^{2}}{4\left(R+r_{+}^{2} / l\right)^{2}}
$$

This is just a negative energy plane wave superimposed on a charged negative mass black string [12] [7].

With the exception of a three dimensional black hole with nonzero rotation, $\partial / \partial t$ is tangent to the horizon for the solutions considered here. Since the horizon is a null surface, the dual with respect to time will be singular where the horizon of the original solution is, when $\partial / \partial t$ is tangent to the horizon. For the three dimensional black hole $\partial / \partial t$ is null at $r=\sqrt{r_{+}^{2}+r_{-}^{2}}$, which is the location of the boundary of its ergosphere. If $J \neq 0$ then this will be outside of the horizon, which is at $r=r_{+}$. To make the dual of the three dimensional black hole more similiar to the other cases one might want to dualize it with respect to the tangent of the horizon.

The vector $\partial / \partial \chi=\partial / \partial t+\frac{r_{-}}{l r_{+}} \partial / \partial \theta$ is tangent to the horizon. The metric (2.6) tells us that $(\partial / \partial \chi)^{2}=-\left(r_{+}^{2}+r_{-}^{2}\right)\left(r^{2}-r_{+}^{2}\right) / l^{2}$, so $\partial / \partial \chi$ is null at the horizon, as it had to be, and timelike outside of it. When $J=0$ this reduces to $\partial / \partial t$. The vector $\partial / \partial \chi$ is a Killing vector so we can dualize the three dimensional black hole with respect to it. Doing this gives an uncharged three dimensional black string with mass per length $-\left(r_{+}^{2}-r_{-}^{2}\right) / l$, as before this is negative. Recall that if the three dimensional black hole had nonzero rotation then dualizing it with respect to $t$ gave a charged negative mass black string.

Like the three dimensional black string, the three dimensional black hole has a spacelike Killing vector, $\partial / \partial \theta$. The results of dualizing the three dimensional black hole with respect to this symmetry are known [7] [8]. The nonextremal case gives us a positive mass 
three dimensional black string, if the black hole has $J \neq 0$ then the black string will have a nonzero charge. The $M=J=0$ extremal case gives a positive energy plane fronted wave and the $J=l M \neq 0$ extremal case gives a plane fronted wave superimposed on an extremally charged black string, both with positive energy. As in the case of the three dimensional black string, except for having negative energies, dualizing a three dimensional black hole with respect to its timelike symmetry gave the same result as dualizing with respect to its spacelike symmetry.

\subsection{Black Strings In Five Or More Dimensions}

Taking $D$ to be the number of spacetime dimensions, the charged black string solutions for $D \geq 5$ are given by [10],

$$
\begin{aligned}
d s^{2}= & -\frac{1-M_{o} / r^{n}}{1+M_{o} \sinh ^{2} \alpha / r^{n}} d t^{2}+\frac{d x^{2}}{1+M_{o} \sinh ^{2} \alpha / r^{n}} \\
& +\frac{d r^{2}}{1-M_{o} / r^{n}}+r^{2} d \Omega_{n+1}^{2} \\
\phi= & -\frac{1}{2} \ln \left(1+\frac{M_{o} \sinh ^{2} \alpha}{r^{n}}\right), \quad B_{t x}=-\frac{M_{o} \cosh \alpha \sinh \alpha}{r^{n}+M_{o} \sinh \alpha}
\end{aligned}
$$

The mass per length of this black string is $M=M_{o}\left(1+n /(n+1) \sinh ^{2} \alpha\right)$, its charge per length is $Q=n /(n+1) \sinh \alpha \cosh \alpha$, and $n$ is related to the spacetime dimension by $n=D-4$. Like the three dimensional black string this solution has translational symmetry and can be constructed in a manner similiar to that of the three dimensional case. These black strings can be constructed by taking the product of a $D-1$ dimensional Schwarzschild black hole with $R$, then boosting with a velocity parameter $\alpha$, and dualizing with respect to $x$ [10]. A $D$ dimensional black string with $\alpha=0$ is the product of a $D-1$ dimensional Schwarzschild black hole and $R$. This tells us that the results we get by dualizing the black strings in $D$ dimensions can be used to get the duals of the $D-1$ black holes by taking $\alpha=0$.

Using (1.3) to dualize (2.12) we find that

$$
\begin{aligned}
\tilde{d s}^{2} & =-\frac{r^{n}+M_{o} \sinh ^{2} \alpha}{r^{n}-M_{o}} d t^{2}-\frac{2 M_{o} \sinh \alpha \cosh \alpha}{r^{n}-M_{o}} d x d t \\
& +\frac{r^{2 n}-r^{n} M_{o}-M_{o}^{2} \sinh ^{2} \alpha \cosh ^{2} \alpha}{\left(r^{n}-M_{o}\right)\left(r^{n}+M_{o} \sinh ^{2} \alpha\right)} d x^{2}+\frac{r^{n}}{r^{n}-M_{o}} d r^{2}+r^{2} d \Omega_{n+1}^{2} \\
\tilde{\phi} & =-\frac{1}{2} \ln \left(1-\frac{M_{o}}{r^{n}}\right), \quad \tilde{B}_{\alpha \beta}=0
\end{aligned}
$$


As expected from the general result, that will be established later, of charge and momentum being interchanged (with a negative sign) we have an uncharged boosted product being the result of dualizing a charged solution that has zero momentum.

If we make the coordinate transformation $t=t^{\prime} \cosh \alpha-x^{\prime} \sinh \alpha$ and $x=x^{\prime} \cosh \alpha-$ $t^{\prime} \sinh \alpha$ the metric becomes

$$
\widetilde{d s}^{2}=\frac{r^{n}}{r^{n}-M_{o}} d t^{\prime 2}+d x^{\prime 2}+\frac{r^{n}}{r^{n}-M_{o}} d r^{2}+r^{2} d \Omega_{n+1}^{2}
$$

This is the product of the dual of a $D-1$ dimensional black hole and a translation. It has mass per length $-(D-5) /(D-3) M_{o}$.

We can get the mass of the dual of a black hole in four or more dimensions from this by taking $D \rightarrow D+1$ and $\alpha=0$. This gives us $\tilde{M}=-(D-4) /(D-2) M_{o}$, where $M_{o}$ is the mass of the original black hole. It is interesting to note that the dual of the usual four dimensional Schwarzschild black hole has zero mass.

The extremal $D \geq 5$ dimensional black string is obtained by taking $M_{o} \rightarrow 0$ and $\alpha \rightarrow \infty$ in such a way that $M_{o} \sinh ^{2} \alpha$ approaches a constant, clearly this gives $M=Q$. If we do this in (2.13) and make the coordinate transformation $u=t+x$ and $v=t-x$ we will get

$$
\widetilde{d s}^{2}=-d u d v-\frac{M_{o} \sinh ^{2} \alpha}{r^{n}} d u^{2}+d r^{2}+r^{2} d \Omega_{n+1}^{2}
$$

This is just a plane fronted wave in $D \geq 5$ dimensions [10]. This solution also has negative energy.

Finally we can compare these duals with the results of dualizing the higher dimensional black string (2.12) with respect to $x$ [10]. Dualizing the nonextremal case with respect to $x$ gives an uncharged boosted positive mass black string that is boosted with a velocity parameter, $\alpha$, implicitly related to the charge per length of the original black string by $Q=n /(n+1) \sinh \alpha \cosh \alpha$. For the extremal case dualizing with respect to $x$ gives a positive energy plane fronted wave, recall that dualizing the extremal solution with respect to $t$ gives a negative energy plane fronted wave.

\section{General Results}

We can get two general results if we consider solutions that are asymptotically flat in the sense that [10] asymptotically we have $g_{\alpha \beta} \rightarrow \eta_{\alpha \beta}+\gamma_{\alpha \beta}, \phi \rightarrow \phi_{o}+\chi, B_{\alpha \beta} \rightarrow B_{\alpha \beta}$ such that $\gamma_{\alpha \beta}, B_{\alpha \beta}$, and $\chi$ all approach zero. We want to allow solutions with translational 
symmetry to be asymptotically flat, so large distances will refer to large transverse distance. The proper distance from the source will be denoted by $r$. If we let $N=D$ for black holes and $N=D-1$ for black strings, then for $N \geq 4$ we require these fields to approach zero at least as fast as $r^{-(3-N)}$. If $D=3$ then the requirement for asymptotic flatness is that they approach zero at least as fast as $e^{-a r}$, with $a$ being some constant. Only the black string solution is relevant here, there do not appear to be asymptotically flat black holes in three dimensions or asymptotically flat black strings in four dimensions. We also require $\phi_{o}=-a r / 2+b$, where $b$ is also a constant. For $D \geq 4 a$ must be zero.

If we take such a solution and apply a duality transformation with respect to a vector that has a norm that approaches a nonzero constant asymptotically, then the dual will also be asymptotically flat. The three dimensional black string is of this form and the three dimensional black hole is not. Even though these solutions are duals of each other [7] [8] (see also section 2.2), there is no conflict because to go from the asymptotically flat solution to the one that is not, one must dualize with respect to a symmetry that is asymptotically null. Given such an asymptotically flat solution and applying (1.3) one finds that to first order the asymptotic parts of the fields transform as

$$
\begin{aligned}
& \tilde{\gamma}_{t t}=-\gamma_{t t}, \quad \tilde{\gamma}_{i j}=\gamma_{i j}, \quad \tilde{\gamma}_{t j}=B_{t j}, \\
& \tilde{B}_{t j}=\gamma_{t j}, \quad \tilde{B}_{i j}=B_{i j}, \quad \tilde{\chi}=\chi+\frac{1}{2} \gamma_{t t}
\end{aligned}
$$

To get our first general result suppose that our spacetime has a spacelike translational symmetry in addition to the timelike one, such as the black string cases. As in these cases call this the $x$ direction. We can calculate the momentum per length in the $x$ direction and the charge of $H_{\alpha \beta \gamma}$ per length by using [10]

$$
\begin{array}{r}
P_{x}=-C \oint e^{-2 \phi}\left(\partial_{r} \gamma_{t x}-\partial_{t} \gamma_{x r}\right) \\
Q=C \oint e^{-2 \phi}\left(\partial_{r} B_{t x}+\partial_{t} B_{x r}\right)
\end{array}
$$

where $C$ is a dimension dependent constant and the integrals are over closed surfaces at large $r$. It has been shown [10 that when we dualize with respect to $x$ we have $\tilde{P}_{x}=Q$ and $\tilde{Q}=P_{x}$. If we want to dualize with respect to $t$ we need $g_{\alpha \beta}, B_{\alpha \beta}$, and $\phi$ all to be independent of $t$. This tells us that the second terms of $P_{x}$ and $Q$ are zero. Therefore, when we dualize with respect to $t$ (3.1) shows us that $\tilde{P}_{x}=-Q$ and $\tilde{Q}=-P_{x}$. Up to a sign this is the same result as dualizing with respect to $x$. 
The formula for the mass of a solution is [10] (more accurately this is a formula for the time component of the momentum, so it is really the energy)

$$
M=C \oint e^{-2 \phi}\left[\nabla^{i} \gamma_{i j}-\partial_{j}(\gamma-4 \chi)-2 \gamma_{i j} \partial^{i} \phi_{o}\right] d S^{j}
$$

where $\gamma$ is the trace of $\gamma_{i j}, \nabla_{i}$ is the covariant derivative associated with the coordinates one chooses at infinity (for example $\nabla_{i}=\partial_{i}$ if we have a Cartesian coordinate system at infinity), and the integral is over a surface at large $r$ - since we are allowing translational symmetries asymptotic flatness refers to large transverse distances from the object [13]. If we dualize with respect to a spacelike symmetry, say $x$, it has been shown that this is invariant [10]. The reason is that the change in $\chi$ under duality is cancelled by that in $\gamma_{x x}$. When we dualize with respect to time we now do not change the spatial metric to first order, but the dilaton still changes. In fact one gets

$$
\tilde{M}=C \oint e^{-2 \phi}\left[\nabla^{i} \gamma_{i j}-\partial_{j}(\gamma-4 \chi)-2 \gamma_{i j} \partial^{i} \phi_{o}+2 \partial_{j} \gamma_{t t}\right] d S^{j}
$$

for the mass of the dual solution. It is easily seen that if one has a solution where $\partial_{r} \gamma_{t t}<0$, then the mass of the dual is less than the mass of the original. This is the second general result.

The condition $\partial_{r} \gamma_{t t}<0$ is basically that we get signals redshifting as they move away from the source. Intuitively one would expect positive mass solutions to exihibit this behavior. However, $\gamma_{t t}$ is the asymptotic part of the string metric and it is really the behavior of the Einstein metric [14] that determines the mass.

If it were true that all positive mass solutions had $\partial_{r} g_{t t}<0$ at infinity then one could show that the dual with respect to time of a positive mass solution has nonpositive mass. The reasoning is as follows. Suppose it were true, then if we were dualizing a positive mass solution we would know that $\partial_{r} \gamma_{t t}<0$, using (3.4) we would then know that $\tilde{M}<M$. Suppose that we also had $\tilde{M}>0$, then if we were to dualize with respect to $t$ again we would then have $\tilde{\tilde{M}}<\tilde{M}$, by using (3.4) again. However we know that dualizing with respect to the same symmetry twice gives one the solution one started with, therefore we know that $\tilde{\tilde{M}}=M$. So by assumming that $\tilde{M}>0$ we have arrived at a contradiction, therefore $\tilde{M}$ must be nonpositive. Thus, if it were true that positive mass solutions always have $\partial_{r} \gamma_{t t}<0$ then we would know that a positive mass solution dualized with respect to time would give a solution with nonpositive mass, or more accurately energy. 
Unfortunately its not clear that all positive mass solutions will have $\partial_{r} \gamma_{t t}<0$. Consider the dual of the four dimensional black hole, it has zero mass and $\partial_{r} \gamma_{t t}=M / r^{2}$, where $M$ is the mass of the black hole that we dualized, so it can take arbitrary values. Suppose we add a small amount of positive mass to this solution. If the mass is small enough its effect on $g_{t t}$ at infinity should be small, but as long as we add a finite amount of positive mass to this we should have a positive mass solution. If this is correct then this would be an example of a spacetime with an asymptotic blueshifting string metric, $\partial_{r} \gamma_{t t}>0$, and a positive mass, leaving us with no general result. More than this, we would now have a positive mass solution that yields another positive mass solution when dualized with respect to time. This follows from the fact that if $\partial_{r} \gamma_{t t}>0$ then (3.4) shows us that $\tilde{M}>M$, so $M>0$ then leads to $\tilde{M}>0$.

\section{Conclusions}

In this work several stationary solutions to the low energy string theory equations were considered. The fact that they have a timelike Killing vector allows one to dualize them with respect to that vector. All of the solutions dualized have horizons and since $\partial / \partial t$ becomes null at, or before, the horizon we expect all of these duals to have naked singularities. For asymptotically flat solutions that have a spacelike symmetry in addition to the timelike one, it was found that dualizing the time exchanged the momentum along the spatial symmetry with the negative charge of the antisymmetric three tensor. Except for the change of sign this is the same result one would get by dualizing the translational symmetry.

All of the examples dualized had positive mass and all of their duals had nonpositive energy. This suggests the possibility of the existence of a general result that this will always be the case. Not only was no general result found, an apparent counterexample to this was described.

Several of the duals gave familiar solutions. The duals of the extremally charged black strings gave negative energy plane fronted waves. The duals of the extremally rotating three dimensional black holes, the $M=J=0$ case and the $J=l M \neq 0$ case, gave negative energy plane fronted waves, in the later case this was superimposed on a negative energy charged black string. The dual of the nonextremal three dimensional black hole is a negative mass black string, if the black hole had nonzero rotation then the black string will be charged. The dual of the three dimensional black string is an uncharged negative 
mass black string. An explanation of this which shows that these solutions correspond to the same conformal field theory was also given.

The fact that these duality transformations map positive mass solutions to ones with negative mass may seem strange because, as stated before, we expect these solutions to be equivalent as string theory solutions. The resolution to this is that we are presumably mapping the exterior region of a solution that has positive mass to a region that has negative mass, but that is only part of a larger space. This being a generalization of the effect of dualizing a two dimensional black hole or a three dimensional black string.

\section{Acknowledgments}

I would like to thank Gary Horowitz for many useful discussions and for reading earlier drafts of this paper. I would also like to thank the Issac Newton Institute for Mathematical Sciences and Cambridge University for providing a stimulating atmosphere while part of the work was being done. This work was supported in part by NSF Grant PHY-9008502. 


\section{References}

[1] K. Kikkawa and M. Yamasaki, Phys. Lett., 149B, 357, 1984.

[2] T. Buscher, Phys. Lett. B201, 466 (1988) ; Phys. Lett. B194, 59 (1987).

[3] M. Roček and E. Verlinde, Nucl. Phys. B373, 630 (1992).

[4] E. Witten, Phys. Rev. D44, 314 (1991).

[5] R. Dijkgraaf, E. Verlinde, and H. Verlinde, Nucl. Phys. B371, 269 (1992).

[6] M. Banados, C. Teitelboim, and J. Zanelli, Phys. Rev. Lett. 69, 1849 (1992); M. Banados, M. Henneaux, C. Teitelboim, and J. Zanelli, Phys. Rev. D48, 1506 (1993).

[7] G. Horowitz and D. Welch, Phys. Rev. Lett. 71, 328 (1993).

[8] N. Kaloper, Phys. Rev. D48, 2598 (1992).

[9] J. Horne and G. Horowitz, Nucl. Phys. B368, 444 (1992).

[10] J. Horne, G. Horowitz, and A. Steif, Phys. Rev. Lett. 68, 568 (1992).

[11] This argument is due to Gary Horowitz.

[12] D. Garfinkle, Phys. Rev. D46, 4286 (1992).

[13] This is a slightly different usage from [10], here the integrals over dimensions with translational symmetries are also being taken.

[14] In dimensions other than two the Einstein metric is obtained by conformally rescaling the string metric by a factor such that the Ricci scalar term in the action is of the usual form, it is given by $g_{\alpha \beta}^{E}=e^{-4 \phi /(D-2)} g_{\alpha \beta}$. 